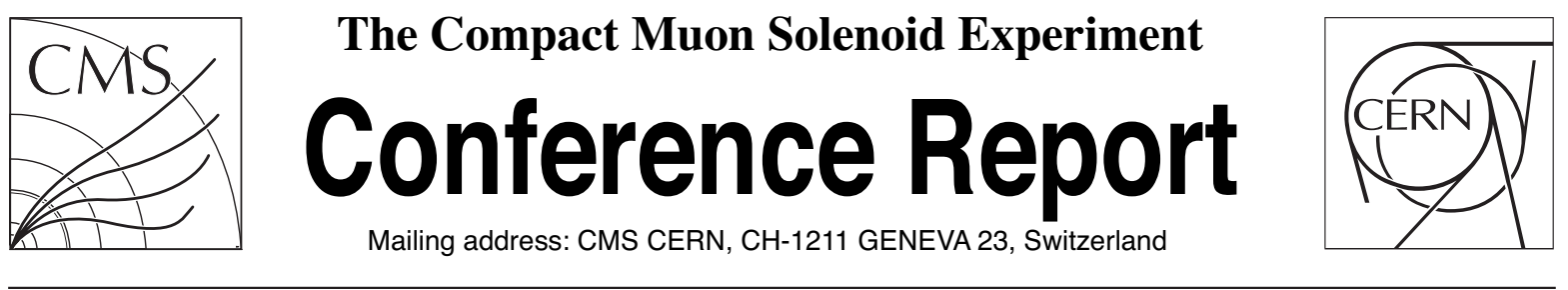

10 February 2020 (v4, 05 August 2021)

\title{
ECAL trigger performance in Run 2 and improvements for Run 3
}

\author{
Davide Valsecchi for the CMS Collaboration
}

\begin{abstract}
The CMS electromagnetic Calorimeter (ECAL) is a high resolution crystal calorimeter operating at the CERN LHC. It is responsible for the identification and precise reconstruction of electrons and photons in CMS, which were crucial in the discovery and subsequent characterization of the Higgs boson. It also contributes to the reconstruction of tau leptons, jets, and calorimeter energy sums, which are are vital components of many CMS physics analyses. The ECAL trigger system employs fast digital signal processing algorithms to precisely measure the energy and timing information of ECAL energy deposits recorded during LHC collisions. These trigger primitives are transmitted to the Level-1 trigger system at the LHC collision rate of $40 \mathrm{MHz}$. These energy deposits are then combined with information from other CMS sub-detectors to determine whether the event should trigger the readout of the data from CMS to permanent storage. This note will summarize the ECAL trigger performance achieved during LHC Run 2 (2015-2018). It will describe the methods that are used to provide frequent calibrations of the ECAL trigger primitives during LHC operation. These are needed to account for radiation-induced changes in crystal and photodetector response and to maintain stable trigger rates and efficiencies up to $|\eta|=3.0$. They also minimize the spurious triggering on direct signals in the photodetectors used in the barrel region $(|\eta|<1.48)$. Both of these effects have increased relative to LHC Run 1 (2009-2012), due to the higher luminosities experienced in Run 2. Further improvements in the energy and time reconstruction of the CMS ECAL trigger primitives are being explored for LHC Run 3 (2021-23), using additional features implemented in the on-detector readout. These are particularly focused on improving the performance at the highest instantaneous luminosities (which will reach or exceed $2 \cdot 10^{34} \mathrm{~cm}^{-2} \mathrm{~s}^{-1}$ in Run 3) and in the most forward regions of the calorimeter $(|\eta|>2.5)$, where the effects of detector aging will be the greatest. The main features of these improved algorithms will be described and preliminary estimates of the expected performance gains will be presented.
\end{abstract}




\section{ECAL trigger performance in Run 2 and improvements for Run 3}

\section{Valsecchi on behalf of the CMS collaboration}

Physics Department “G. Occhialini”, Università degli Studi di Milano-Bicocca, Piazza della Scienza 3, Milano, Italy

E-mail: davide.valsecchi@cern.ch

AвstRact: The CMS electromagnetic Calorimeter (ECAL) is a high resolution crystal calorimeter operating at the CERN LHC. It is responsible for the identification and precise reconstruction of electrons and photons in CMS, which were crucial in the discovery and subsequent characterization of the Higgs boson. It also contributes to the reconstruction of tau leptons, jets, and calorimeter energy sums, which are vital components of many CMS physics analyses.

The ECAL trigger system employs fast digital signal processing algorithms to precisely measure the energy and timing information of ECAL energy deposits recorded during LHC collisions. These trigger primitives are transmitted to the Level-1 trigger system at the LHC collision rate of $40 \mathrm{MHz}$. These energy deposits are then combined with information from other CMS sub-detectors to determine whether the event should trigger the readout of the data from CMS to be transmitted to the High-Level Trigger and eventually to permanent storage.

This note will summarize the ECAL trigger performance achieved during LHC Run 2 (20152018). It will describe the methods that are used to provide frequent calibrations of the ECAL trigger primitives during LHC operation. These are needed to account for radiation-induced changes in crystal and photodetector response and to maintain stable trigger rates and efficiencies up to $|\eta|=3.0$. They also minimize the spurious triggering on direct signals in the photodetectors used in the barrel region $(|\eta|<1.48)$. Both of these effects have increased relative to LHC Run 1 (2009-2012), due to the higher luminosities experienced in Run 2.

Further improvements in the energy and time reconstruction of the CMS ECAL trigger primitives are being explored for LHC Run 3 (2021-2023), using additional features implemented in the on-detector readout. These are particularly focused on improving the performance at the highest instantaneous luminosities (which will reach or exceed $2 \cdot 10^{34} \mathrm{~cm}^{-2} \mathrm{~s}^{-1}$ in Run 3) and in the most forward regions of the calorimeter $(|\eta|>2.5)$, where the effects of detector aging will be the greatest. The main features of these improved algorithms will be described and preliminary estimates of the expected performance gains will be presented.

Keywords: Calorimeters; Performance of High Energy Physics Detectors; Trigger algorithms; Trigger concepts and systems (hardware and software) 


\section{Contents}

1 Introduction 1

1.1 The CMS Electromagnetic Calorimeter (ECAL) 1

1.2 The CMS trigger 1

1.3 The ECAL Trigger Primitive Generation (TPG) 2

2 Trigger performance in Run $2 \quad 2$

3 Optimization for Run $3 \quad 3$

3.1 Weights granularity 4

3.2 Optimization for PU 4

3.3 Further studies 5

4 Conclusions 6

\section{Introduction}

\subsection{The CMS Electromagnetic Calorimeter (ECAL)}

The CMS electromagnetic calorimeter (ECAL) [1] is a homogeneous calorimeter made of 75848 lead tungstate $\left(\mathrm{PbWO}_{4}\right)$ scintillating crystals, located inside the CMS [2] superconducting solenoid magnet. It is made of a barrel part (EB) covering the region of pseudorapidity $|\eta|<1.48$ with 61200 crystals and two endcaps (EE), which extend the coverage up to $|\eta|<3.0$ with 7324 crystals each. Scintillation light is detected with avalanche photodiodes (APD) in the barrel and vacuum phototriodes (VPT) in the endcaps. The ECAL is crucial for the identification and reconstruction of photons and electrons, and the measurement of jets and of missing transverse momentum. The electrons and photons are typically reconstructed up to $|\eta|<2.5$, the region covered by the tracker, while jets are reconstructed up to $|\eta|<3.0$.

\subsection{The CMS trigger}

Given the high rate of collisions in CMS, one bunch crossing every $25 \mathrm{~ns}$, it is not possible to save information from all events and a trigger system is implemented to identify and read-out only interesting physics events. The CMS trigger is implemented as a two-stage system. The Level-1 trigger (L1) consists of custom hardware that elaborates coarse-grained information from various detector subsystems in order to read-out events at a rate below $100 \mathrm{kHz}$ [3]. The $\mathrm{L} 1$ trigger needs to deliver a decision within a latency of a few $\mu$ s because the full-granularity information for each event is kept in on-detector electronic for a limited time. The second level, the High Level Trigger (HLT), is implemented in software and performs a full reconstruction of the event using more granular detector information to reduce the event rate to $\sim 1 \mathrm{kHz}$, which is written to disk. 
ECAL produces trigger primitives (TPs) every $25 \mathrm{~ns}$ that are sent to the L1 trigger system to form electrons, photons, jet candidates, and to compute transverse energy sums. These TPs comprise a transverse energy measurement for a matrix of crystals, with one or two feature bits that characterise the energy spread between crystals within the TP, and a bunch crossing (BX) assignment.

\subsection{The ECAL Trigger Primitive Generation (TPG)}

The ECAL TPs are computed on-detector by custom ASICs (named FENIX [4]) located on the Front End (FE) boards. The analog signals from photodetectors in EB and EE are amplified, sampled, digitized, and calibrated by custom ASICs located on Very Front End (VFE) boards (see figure 1, left). Each VFE card amplifies and digitises the signals from up to 5 channels, referred to as a "strip". The FE cards process the data and compute the TPs from up to 5 strips. Then, the sampled pulses for each strip are summed and a Finite Impulse Response (FIR) filter is applied to estimate the signal amplitude as a weighted sum of the samples around the signal peak (see figure 1, right). The weights are configurable, and are assigned to the Fenix registers by the data acquisition system (DAQ). Since the digital filter is applied in a sliding time window, the energy is finally assigned to the correct BX applying a simple peak finder algorithm. Finally, the strip fine grain veto bit (sFGVB), is computed for barrel TPs to reject anomalous signals in the barrel APDs [5].
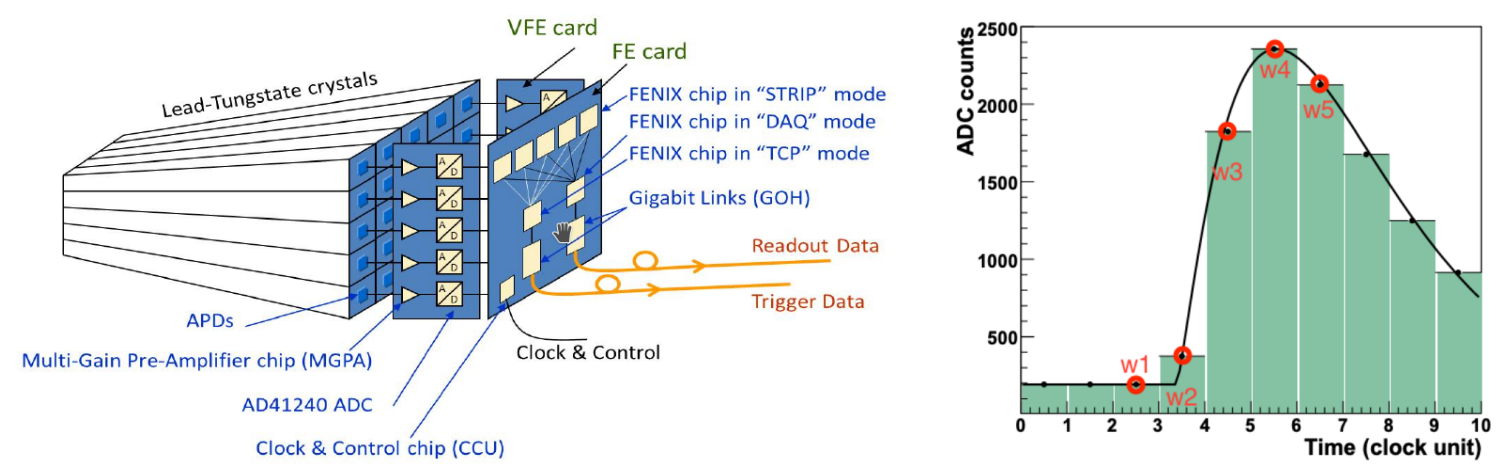

Figure 1. Left: a sketch of the ECAL front-end readout electronics. Right: the application of the weights filter on the ECAL signal pulse shape. In this example, 5 non-zero weights are applied.

\section{Trigger performance in Run 2}

During Run 2 of the LHC (2015-2018) a greater instantaneous and total integrated luminosity was reached compared to Run 1 (2011-2012). Radiation-induced effects created challenging data taking conditions for ECAL and frequent calibration of the parameters of the ECAL trigger system were necessary to maintain an optimal trigger efficiency and a stable TP energy scale.

Multiple time dependent corrections were applied during Run 2 to account for radiation-induced effects: laser response corrections, crystal inter-calibration, pedestal drift corrections, and pulse shape corrections. As a result of these calibrations and corrections, excellent L1 reconstruction efficiencies for electron and photon candidates have been maintained throughout Run 2 [6] as shown in figure 2. 
Laser response corrections. As ECAL sustains more radiation from LHC collisions, the crystal transparency is reduced, and the light output measured by the photodetectors for a given deposited energy decreases. The loss of transparency is small in EB, about 10\%, but it is more noticeable as $\eta$ increases, along with radiation dose, in EE, as shown in figure 3, left. This effect is compensated by the ECAL laser monitoring system: a reference laser light is shone onto all crystals during one orbit gap (calibration gap) of the LHC orbit to calibrate the crystal light output response. The calibration constants are then computed during the offline reconstruction [7]. These calibration factors have been propagated to the L1 trigger and HLT twice a week during Run 2 to keep the TP energy scale stable [8].

Pedestal drift corrections. In EB, particles can directly ionize the barrel APD, leading to anomalous isolated signals [9]. These signals, called spikes, occur at a rate directly proportional to the instantaneous luminosity of LHC and must be suppressed at L1 to avoid wasting trigger bandwidth. They are identified on-detector looking for isolated signals over a configurable energy threshold and such TPs are prevented from triggering CMS. The spike killer performance is dependent on the pedestal, the mean background level that must be measured and updated periodically during the data taking to maintain optimal efficiency. Figure 3 on the right shows the improvement in spike rejection due to pedestal updates.

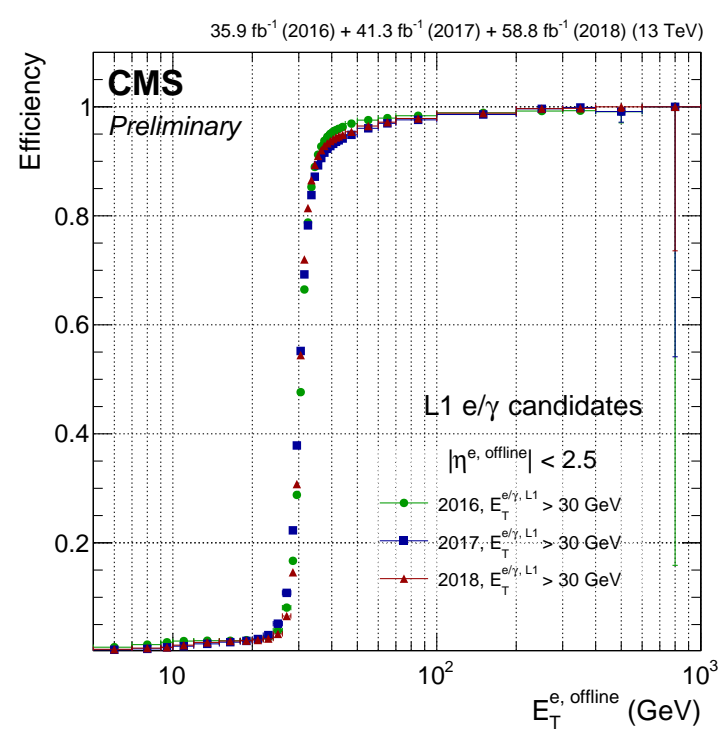

Figure 2. Level-1 Trigger efficiency turn-on curve for electron and photon candidates along Run 2 [6].

\section{Optimization for Run 3}

One set of weights for EB and one for EE are currently used by the on-detector FENIX chips to estimate the TP signal amplitudes. These weights were derived using the ideal pulse shape for undamaged crystals measured during the ECAL test beams. The weights have been used throughout Run 1 and Run 2 without changes. These weights are no longer optimal because of radiation-induced changes to the pulse shapes, especially at high $\eta$ in EE, that induce large biases in the TP energy 

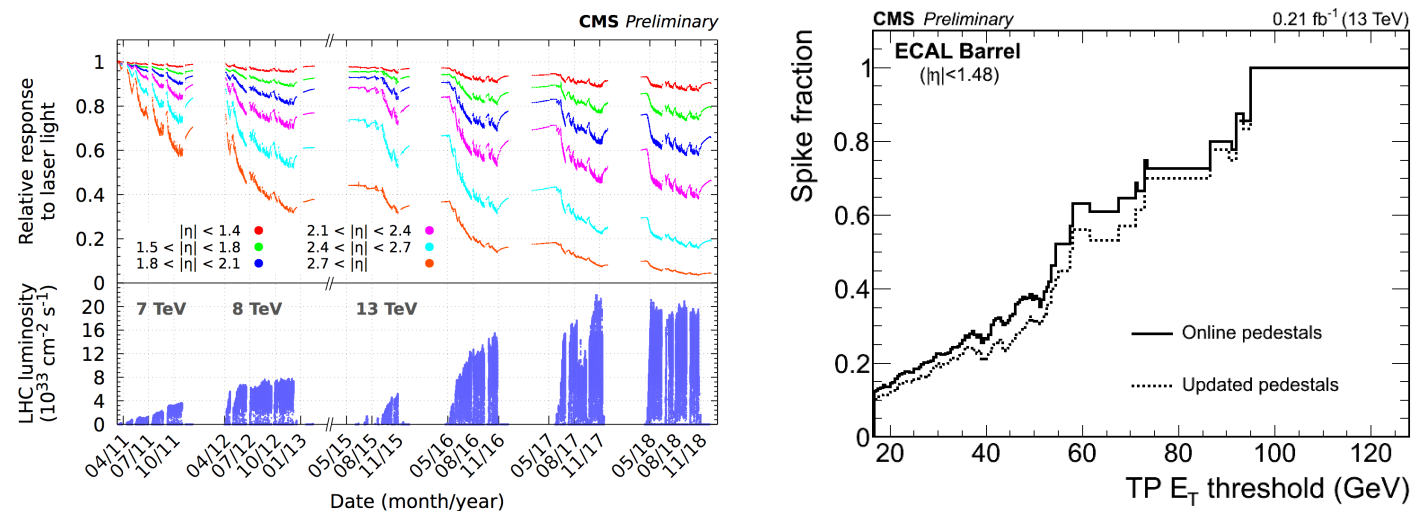

Figure 3. Left: history of ECAL crystals transparency during Run 1 and Run 2 of LHC. Right: the effect of pedestal updates on the efficiency of anomalous signals rejection in the ECAL barrel.

estimate. To keep the excellent trigger performance reached so far and cope with the further pulse shape distortions due to detector ageing and increase out-of-time pileup (PU) foreseen during Run 3, a re-optimization of the Trigger Primititive Generation (TPG) weights will be performed.

\subsection{Weights granularity}

The TPG weights are applied on-detector on a strip-by-strip basis, where a strip is a grouping of 5 nearby crystals. This is the maximal granularity that can be reached in the weights optimization and application. Figure 4 shows the bias in the amplitude reconstruction, defined as $\left(E_{T P} / E_{\text {true }}\right)-1$ where $E_{\text {true }}$ and $E_{T P}$ are the true and reconstructed amplitudes respectively, for different weights configurations considering a signal-only scenario (no out-of-time PU) in the endcaps. The violet line shows the large positive bias created by the current weights due to the radiation-induced changes in pulse shapes. The blue line shows the improvement obtained by using updated pulse shapes, precisely measured for each crystal in September 2018 at the end of Run 2 shifting the acquisition time by small steps and fitting the pulse shape, to obtain one averaged set of weights for EE. Increasing the granularity of the weights up to the strip level (red line) decreases further the bias in the energy reconstruction [10].

\subsection{Optimization for $\mathbf{P U}$}

The ECAL trigger uses 5 pulse samples (as shown in figure 1) to reconstruct the amplitude, therefore it can be biased by PU interactions happening before or after the signal BX, called out-of-time (OOT) PU. The effect of OOT PU varies along $\eta$ and it is more pronounced in the innermost rings of the endcaps.

Moreover, the position of the signal within LHC proton bunch trains changes the average OOT PU effect on the pulse shape. For example, the LHC schema in 2017, trains with 8 colliding proton bunches followed by 4 empty ones ( $8 \mathrm{~b} 4 \mathrm{e}$ ), with continuously varying OOT PU is the most challenging for the ECAL trigger.

A standalone MC simulation has been developed to model the ECAL digitized samples for different pulse shapes, signal energies, pileup, and LHC train structures. With this setup it is possible to derive optimal weights for various data-taking conditions and to study the effect of 


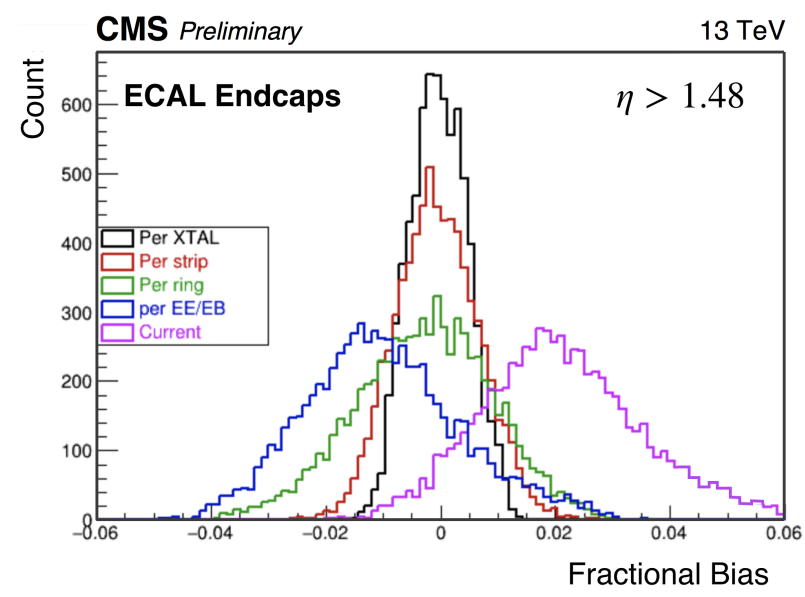

Figure 4. Fractional bias in amplitude reconstruction $\left(E_{T P} / E_{\text {true }}\right)-1$ for current weights (violet), updated pulse shapes weights (blue), and weights optimized with finer granularity: $\eta$ ring (green), strip (red), and crystal (black).

different sets of weights on configurable scenarios. Two sets of weights have been derived for each strip simulating the PU level expected for Run $3(\langle P U\rangle \sim 50)$ : one set optimized for low energy signal TPs $\left(E_{T}=2 \mathrm{GeV}\right)$ and the other for high energy ones $\left(E_{T}=30 \mathrm{GeV}\right)$. The performance of these sets of weights has been evaluated in terms of amplitude reconstruction bias (average $\left.\left(E_{\mathrm{TP}} / E_{\text {true }}\right)-1\right)$ and fractional spread $\left(\mathrm{RMS}\left[E_{\mathrm{TP}} / E_{\text {true }}\right] /\left(E_{\mathrm{TP}} / E_{\text {true }}\right)\right)$ both in simulation and by re-emulating the trigger primitives on 2017 and 2018 CMS data using events selected randomly to avoid bias [11].

Figure 5 (left), shows the TP fractional spread plotted as a function of signal transverse energy, analyzed in the high $\eta$ region of the endcaps applying different sets of weight on CMS data from 2017 ( $\langle P U\rangle \sim 40$ and LHC schema 8b4e). Run 2 current weights (orange line) are compared with PU optimized weights for low and high energy (respectively green and blue line), with updated weights averaged on EE (light blue line), and with weights optimized by strip at $\mathrm{PU}=0$ (red line). There is a strong $E_{T}$ dependence to the TP bias and resolution, especially at low energy TPs, and the PU optimized weights for $E_{T}=2 \mathrm{GeV}$ signals give a measurable resolution improvement.

There is also a strong bunch position dependence to the TP resolution as shown in figure 5, right. Using PU optimized weights computed for low energy signals (green line) significantly improves the resolution and reduces variations along the LHC trains. The PU optimization improves the average behaviour, but cannot account completely for pulse-to-pulse and BX-to-BX distortions.

\subsection{Further studies}

It is known that the FENIX chips used to compute the TPs in the ECAL front-end are capable of using an additional 6th weight for the amplitude reconstruction. They also contain a second filter with a separate set of weights, which have never been used in ECAL trigger. The interplay of the two filters has a limited set of operation modes: e.g. the output TP can be the sum of the two filters, or one filter can be used to veto the other only if its output is greater. The possible improvements from these unused features of the electronics are currently under study: there is the potential to use 

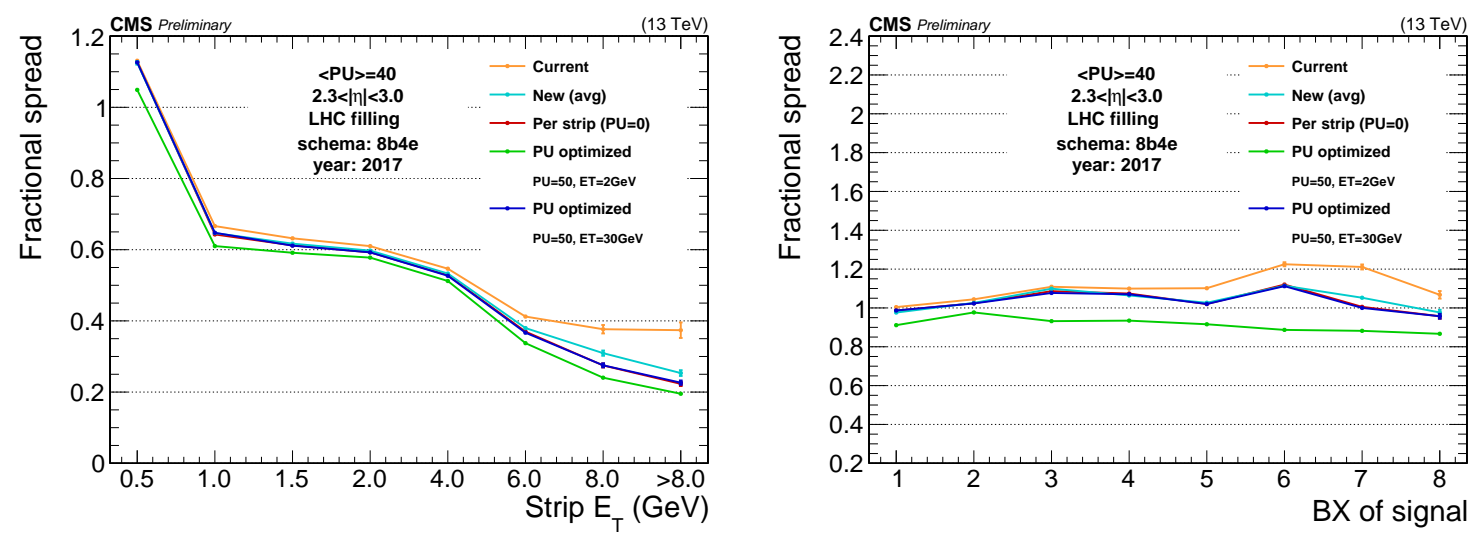

Figure 5. Left: fractional spread, or TP resolution, versus signal $E_{T}$ per strip, in the high $\eta$ rings of the ECAL endcaps. Right: fractional spread by BX position of the signal along the LHC train. Different sets of weights are re-emulated on CMS data from 2017, corresponding to $\langle P U\rangle \sim 40$ and the 8b4e LHC schema [11].

the second filter to perform a timing estimation of the pulse in order to improve the spike rejection in the barrel, or to identify and veto TPs with large OOT PU contributions.

\section{Conclusions}

The ECAL trigger system employs fast digital signal processing algorithms to precisely measure the energy and timing information of ECAL energy deposits recorded during LHC collisions. During Run 2, as a consequence of constant detector monitoring and periodic calibrations, the ECAL trigger has been able to cope with increased integrated luminosity and PU and to maintain excellent L1 trigger efficiency for electrons and photons.

For Run 3 of the LHC, studies are being performed with the goal to fully exploit the ECAL trigger electronics capabilities. Amplitude reconstruction weights have been re-optimized increasing their granularity and including the effect of PU, and the improvements in TP resolution and bias have been shown in data and simulation. Finally, unused features of the electronics, including an additional 6th weight for amplitude reconstruction and a second filter, are being explored and their ability to improve the ECAL trigger will be fully assessed.

\section{Acknowledgments}

Special thanks to David A. Petyt, Abraham T. Charny, and the whole CMS Collaboration.

\section{References}

[1] CMS collaboration, The CMS electromagnetic calorimeter project: technical design report, CERN-LHCC-97-033, CERN, Geneva, Switzerland (1997) [CMS-TDR-4].

[2] CMS collaboration, The CMS experiment at the CERN LHC, 2008 JINST 3 S08004.

[3] A. Zabi et al., The CMS level-1 calorimeter trigger for the LHC Run II, 2017 JINST 12 C01065. 
[4] M. Hansen, CMS ECAL FENIX ASIC design methodology, CERN-2004-010, CERN, Geneva, Switzerland (2004).

[5] P. Adzic et al., Reconstruction of the signal amplitude of the CMS electromagnetic calorimeter, Eur. Phys. J. C 46 (2006) 23.

[6] CMS Collaboration, Level-1 E/Gamma trigger performance in Run II, CERN-CMS-DP-2019-020, CERN, Geneva, Switzerland (2019).

[7] M. Anfreville et al., Laser monitoring system for the CMS lead tungstate crystal calorimeter, Nucl. Instrum. Meth. A 594 (2008) 292.

[8] CMS collaboration, CMS ECAL response to laser light, CMS-DP-2019-005, CERN, Geneva, Switzerland (2019).

[9] CMS collaboration, Mitigation of anomalous APD signals in the CMS electromagnetic calorimeter, $J$. Phys. Conf. Ser. 404 (2012) 012043.

[10] CMS collaboration, CMS ECAL trigger plots, CERN-CMS-DP-2019-031, CERN, Geneva, Switzerland (2019).

[11] CMS collaboration, Performance of a standalone simulation and potential improvements of the ECAL trigger primitive algorithms using LHC Run 2 data, CERN-CMS-DP-2019-040, CERN, Geneva, Switzerland (2019). 